\title{
Damping of elementary excitations in one-dimensional dipolar Bose gases
}

\author{
Hadrien Kurkjian (1) \\ TQC, Universiteit Antwerpen, Universiteitsplein 1, 2610 Antwerpen, Belgium \\ Zoran Ristivojevic (1) \\ Laboratoire de Physique Théorique, Université de Toulouse, CNRS, UPS, 31062 Toulouse, France
}

(Received 12 March 2020; accepted 2 July 2020; published 31 August 2020)

\begin{abstract}
In the presence of dipolar interactions, the excitation spectrum of a Bose gas can acquire a local minimum. The corresponding quasiparticles are known as rotons. They are gapped and do not decay at zero temperature. Here we study the decay of rotons in one-dimensional Bose gases at low temperatures. It predominantly occurs due to the backscattering of thermal phonons on rotons. The resulting rate scales with the third power of temperature and is inversely proportional to the sixth power of the roton gap near the solidification phase transition. The hydrodynamic approach used here enables us to find the decay rate for quasiparticles at practically any momenta, with minimal assumptions on the exact form of the interparticle interactions. Our results are an essential prerequisite for the description of all the dissipative phenomena in dipolar gases and have direct experimental relevance.
\end{abstract}

DOI: 10.1103/PhysRevResearch.2.033337

\section{INTRODUCTION}

At low pressures and temperatures, helium-4 is a remarkable quantum liquid that is superfluid. Landau characterized the latter state by a dissipationless flow of macroscopic objects at low velocities [1]. Another particular feature of the superfluid helium is seen in its spectrum of elementary excitations. While at the lowest momenta it is linear, the spectrum possesses a local minimum. The corresponding quasiparticles are known as rotons and have wavelengths that practically coincide with the mean interparticle distance. Since the interaction between helium atoms is strong, the roton can be visualized as a yet undeveloped Goldstone mode due to an instability toward crystallization [2]. However, such a so-called supersolid state that unifies superfluidity with crystalline order has not been observed in helium, despite some controversies [3-5].

Another system that shows some similarities to superfluid helium are dipolar Bose gases, which can be realized with atoms possessing large dipolar moments, such as chromium, dysprosium, and erbium. Bose-Einstein condensates of those atoms have been realized [6-9], which opened new avenues for studying various phenomena that originate from the dipolar interaction [10,11]. Examples include striped states [12], quantum droplets $[13,14]$, and the elusive supersolid state [15-20].

Trapped dipolar Bose gases can exhibit a quasiparticle spectrum with a roton minimum [21,22]. This occurs

Published by the American Physical Society under the terms of the Creative Commons Attribution 4.0 International license. Further distribution of this work must maintain attribution to the author(s) and the published article's title, journal citation, and DOI. because the dipolar interaction cannot be described only by a short-range pseudopotential, but it must also include an anisotropic long-range part in order to correctly describe the low-energy scattering between bosons [11]. The quasiparticle spectrum in weakly interacting Bose gases is determined by the Bogoliubov theory [23] and depends on the Fourier transform of the pseudopotential. Since it is described by the two parameters, one for the short-range part and the other for the long-range part, when they are properly tuned, the local minimum can develop in the spectrum. A recent experiment [24] has confirmed the presence of rotons in the dipolar Bose gas.

The current understanding of the properties and the dynamics of dipolar gases is limited due to the lack of dissipative mechanisms in most theoretical descriptions. Without dissipation, one can neither describe the postquench relaxation observed in Ref. [24] and the loss of phase coherence [25,26] nor predict the thermodynamic quantities related to ergodicity, such as the gas viscosity [27,28]. In a well-isolated gas, dissipation arises primarily from the interactions between the quasiparticles, which allow the system to reach equilibrium. A prerequisite to understand the dissipative dynamics is thus to compute the quasiparticle lifetime, which is the purpose of this work.

At zero temperature, rotons and all quasiparticle excitations at lower momenta in a dipolar Bose gas are stable. Being slower than the sound velocity, those quasiparticles cannot emit phonons due to the conservation laws of momentum and energy. Such a scenario resembles the absence of Cherenkov radiation at small velocities. However, the quasiparticles do decay at finite temperature. The dominant process for the damping of a subsonic quasiparticle involves its scattering with a thermally excited quasiparticle, where two new 


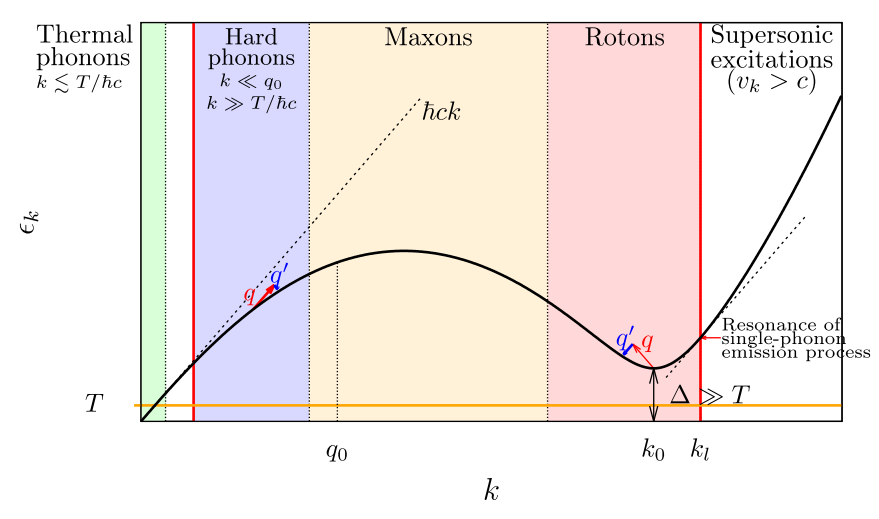

FIG. 1. Rotonic excitation spectrum of a one-dimensional dipolar Bose gas obtained from the Bogoliubov theory [35]. The damping of energetic excitations of the energy $\epsilon_{k} \gg T$, i.e., hard phonons, maxons, and rotons, is controlled by the backscattering off thermal phonons. Our theory is valid until quasiparticles become supersonic with velocities $v_{k}>c$ (right vertical red line at $k_{l}$ ).

quasiparticles are created [27,29-33]. The resulting rate typically scales as a power law of temperature.

In this paper we study the damping of energetic quasiparticles (including rotons) in a one-dimensional dipolar Bose gas. This process is controlled by the backscattering of thermal phonons. We find a low-temperature rate that scales as the third power of temperature $T^{3}$. The hydrodynamic approach employed here to describe the interaction between phonons and energetic quasiparticles is not limited to the weak-coupling regime and does not require a specific form of the interaction potential. It is an important generalization to arbitrary high-energy quasiparticles of the phonon-roton hydrodynamic approach [27]. To describe roton damping, we simply require specific interactions that lead to the formation of a roton minimum. Near the solidification phase transition, when the roton gap $\Delta$ is much smaller than the other relevant energy scales (except temperature), we find a rate diverging as $1 / \Delta^{6}$. Our results pave the way for a description of the postquench relaxation dynamics of a dipolar Bose gas [34].

\section{DAMPING OF ENERGETIC QUASIPARTICLES}

We consider a gas of bosons with a dipolar interaction between particles in a (quasi-)one-dimensional geometry (as studied theoretically in Ref. [35] and recently realized experimentally [24]). We assume that the gas is prepared at low temperature,

$$
T \ll \Delta, m c^{2}
$$

where $m c^{2}$ is the characteristic energy scale for phonons, $m$ denotes the mass of particles, $c$ is the sound velocity, and the Boltzmann constant is set to unity. Subsonic quasiparticles with dispersion $\epsilon_{k}$ are characterized by the velocity $v_{k}=$ $d \epsilon_{k} / \hbar d k$, which is smaller than $c$. They can decay only due to scattering off thermally excited quasiparticles. At very high momenta, the quasiparticles become supersonic (see Fig. 1). In this case they can decay at $T=0$ by emitting phonons, which is not precluded by the conservation laws $[36,37]$.
The collisionless damping rate ${ }^{1}$ associated with a subsonic energetic quasiparticle of the energy $\epsilon_{k} \gg T$ (see Fig. 1) can be computed using the Fermi golden rule and the Boltzmann equation. It leads to

$$
\Gamma_{k}=\frac{2 \pi}{\hbar} \sum_{q, q^{\prime}, k^{\prime}} \frac{\left|\mathcal{A}_{i f}\right|^{2}}{L^{2}} \delta\left(\epsilon_{k}+\hbar \omega_{q}-\epsilon_{k^{\prime}}-\hbar \omega_{q^{\prime}}\right) n_{q}\left(1+n_{q^{\prime}}\right) .
$$

Here $\omega_{q}=c|q|$ is the phonon frequency, $L$ is the system size, and $n_{q}=1 /\left[\exp \left(\hbar \omega_{q} / T\right)-1\right]$ defines the Bose occupation factor. In Eq. (2), $\mathcal{A}_{i f}$ is the transition amplitude from the initial state $|i\rangle=\hat{\gamma}_{k}^{\dagger} \hat{b}_{q}^{\dagger}|0\rangle$, with an excitation present in the mode $k$, to the final state $|f\rangle=\hat{\gamma}_{k^{\prime}}^{\dagger} \hat{b}_{q^{\prime}}^{\dagger}|0\rangle$. Denoted by $\hat{b}^{\dagger}$ and $\hat{\gamma}^{\dagger}$ are the bosonic creation operators for the phonon and the energetic quasiparticle, respectively. The $\delta$ function in Eq. (2) accounts for the energy conservation. Note that in the low-temperature regime (1) considered here, the thermal population of the final roton state is exponentially suppressed, $n_{k^{\prime}} \approx e^{-\epsilon_{k^{\prime}} / T} \ll 1$, and the rate acquires the simple form (2).

The transition amplitude $\mathcal{A}_{i f}$ can be computed with minimal assumptions, in particular, not assuming that the gas is weakly interacting, using quantum hydrodynamics $[27,30,32,33]$. Within this theory, it is sufficient to consider the cubic residual interaction among phonons given by the Hamiltonian $^{2}$

$$
\hat{H}_{\mathrm{ph}}=\sum_{q} \hbar \omega_{q} \hat{b}_{q}^{\dagger} \hat{b}_{q}+\sum_{q, q^{\prime}} \frac{\mathcal{A}_{3}\left(q, q^{\prime}\right)}{\sqrt{L}}\left(\hat{b}_{q+q^{\prime}}^{\dagger} \hat{b}_{q} \hat{b}_{q^{\prime}}+\text { H.c. }\right) .
$$

The matrix element $\mathcal{A}_{3}\left(q, q^{\prime}\right)$ follows from the hydrodynamic equations of motion $[27,38]$ and has the form

$$
\begin{aligned}
\mathcal{A}_{3}\left(q, q^{\prime}\right)= & \frac{m c^{2}}{\sqrt{32 \rho}} \sqrt{\frac{q q^{\prime}\left(q+q^{\prime}\right)}{q_{0}^{3}}}\left[\frac{\rho^{2}}{c^{2}} \frac{d}{d \rho}\left(\frac{c^{2}}{\rho}\right)+\operatorname{sgn}\left(q q^{\prime}\right)\right. \\
& \left.+\operatorname{sgn}\left[q\left(q+q^{\prime}\right)\right]+\operatorname{sgn}\left[q^{\prime}\left(q+q^{\prime}\right)\right]\right],
\end{aligned}
$$

where $\rho$ is the (mean) fluid density and $q_{0}=m c / \hbar$. The hydrodynamics describes the energetic quasiparticles perturbed by the phonon field within a local density approximation as

$$
\hat{H}_{\mathrm{qp}}=\frac{1}{2}[\epsilon(\hat{p}, \rho+\delta \hat{\rho}(\hat{r}))+\hat{p} \hat{v}(\hat{r})+\text { H.c. }] .
$$

Here $\epsilon(\hat{p}, \rho)$ is the Hamiltonian of the unperturbed quasiparticle in first quantization, where $\hat{r}$ is its position and $\hat{p}$ its momentum operator. By $\delta \hat{\rho}$ and $\hat{v}$ are denoted, respectively, the density and the superfluid velocity perturbations caused by the phonons. Expanded to a quadratic order at small $\delta \hat{\rho} \ll \rho$

\footnotetext{
${ }^{1}$ The thermal phonons of the characteristic wave vector $q_{\mathrm{th}}=T / \hbar c$ have a lifetime that diverges when $T \rightarrow 0$. Therefore, $\epsilon_{k} \gg \hbar \Gamma_{q_{\mathrm{th}}}$, which justifies the collisionless approximation.

${ }^{2} \mathrm{We}$ omit the vacuum cubic terms of the form $\hat{b}_{\mathbf{q}} \hat{b}_{\mathbf{q}^{\prime}} \hat{b}_{-\mathbf{q}-\mathbf{q}^{\prime}}$ in Eq. (3). Since they describe far-off-shell processes, those terms do not contribute to the on-shell phonon-quasiparticle scattering amplitude (8) to second-order perturbation theory.
} 
and expressed in second quantization, Eq. (5) becomes

$$
\begin{aligned}
\hat{H}_{\mathrm{qp}}= & \sum_{k} \epsilon_{k} \hat{\gamma}_{k}^{\dagger} \hat{\gamma}_{k}+\sum_{k, q} \frac{\mathcal{A}_{1}(k, q)}{\sqrt{L}}\left(\hat{\gamma}_{k+q}^{\dagger} \hat{\gamma}_{k} \hat{b}_{q}+\text { H.c. }\right) \\
& +\sum_{k, q, q^{\prime}} \frac{\mathcal{A}_{2}\left(k, q, q^{\prime}\right)}{L} \hat{\gamma}_{k+q-q^{\prime}}^{\dagger} \hat{b}_{q^{\prime}}^{\dagger} \hat{\gamma}_{k} \hat{b}_{q} .
\end{aligned}
$$

The phonons and the energetic quasiparticle are coupled by

$$
\begin{aligned}
\mathcal{A}_{1}(k, q) & =\sqrt{\frac{\rho|q|}{2 q_{0}}} \frac{\partial_{\rho} \epsilon_{k}+\partial_{\rho} \epsilon_{k+q}}{2}+\sqrt{\frac{\hbar^{3} c}{2 m \rho|q|}} q\left(k+\frac{q}{2}\right), \\
\mathcal{A}_{2}\left(k, q, q^{\prime}\right) & =\frac{\rho \sqrt{\left|q q^{\prime}\right|}}{2 q_{0}} \frac{\partial_{\rho}^{2} \epsilon_{k}+\partial_{\rho}^{2} \epsilon_{k+q-q^{\prime}}}{2} .
\end{aligned}
$$

Computing the transition amplitude in second-order perturbation theory (including the contribution of the three-phonon residual interaction $[39,40]$ ), on the mass shell we obtain

$$
\begin{aligned}
\mathcal{A}_{i f}= & {\left[\mathcal{A}_{2}\left(k, q, q^{\prime}\right)+\frac{\mathcal{A}_{1}\left(k-q^{\prime}, q^{\prime}\right) \mathcal{A}_{1}\left(k-q^{\prime}, q\right)}{\epsilon_{k^{\prime}}-\hbar \omega_{q}-\epsilon_{k^{\prime}-q}}\right.} \\
& +\frac{\mathcal{A}_{1}(k, q) \mathcal{A}_{1}\left(k^{\prime}, q^{\prime}\right)}{\hbar \omega_{q}+\epsilon_{k}-\epsilon_{k+q}}+\frac{2 \mathcal{A}_{3}\left(q^{\prime}, q-q^{\prime}\right) \mathcal{A}_{1}\left(k, q-q^{\prime}\right)}{\hbar \omega_{q}-\hbar \omega_{q-q^{\prime}}-\hbar \omega_{q^{\prime}}} \\
& \left.+\frac{2 \mathcal{A}_{3}\left(q, q^{\prime}-q\right) \mathcal{A}_{1}\left(k^{\prime}, q^{\prime}-q\right)}{\epsilon_{k}-\epsilon_{k^{\prime}}-\hbar \omega_{q-q^{\prime}}}\right] \delta_{k+q, k^{\prime}+q^{\prime}}
\end{aligned}
$$

To compute the damping rate (2) at low temperature, we need the amplitude (8) expressed at the leading order in small $q$ and $q^{\prime}$. The energy conservation constraint of Eq. (2) has nontrivial solutions only for the backscattering events, i.e., at $q q^{\prime}<0$, which leads to the relation

$$
q^{\prime}=-q \times \begin{cases}\left(c-v_{k}\right) /\left(c+v_{k}\right)+O(q), & k q>0 \\ \left(c+v_{k}\right) /\left(c-v_{k}\right)+O(q), & k q<0 .\end{cases}
$$

Since the quasiparticle scattering off a phonon experiences a small energy change, we expand it to second order around the initial energy $\epsilon_{k}$ as

$\epsilon_{k^{\prime}}(\rho)=\epsilon_{k}(\rho)+\hbar v_{k}(\rho)\left(k^{\prime}-k\right)+\frac{\hbar^{2}\left(k^{\prime}-k\right)^{2}}{2 m^{*}(k, \rho)}+O\left(k^{\prime}-k\right)^{3}$.

In the limit $q \rightarrow 0$, the on-shell amplitude takes the form $\mathcal{A}_{i f}=\hbar c \sqrt{\left|q q^{\prime}\right|} Y_{k} \delta_{k+q, k^{\prime}+q^{\prime}} / 2 \rho$, where

$$
\begin{aligned}
Y_{k}= & \frac{\rho^{2}}{m c^{2}\left(c^{2}-v_{k}^{2}\right)}\left[\left(c^{2}-v_{k}^{2}\right) \partial_{\rho}^{2} \epsilon_{k}-\left(\partial_{\rho} \epsilon_{k}\right) \partial_{\rho}\left(c^{2}-v_{k}^{2}\right)\right. \\
& \left.+\frac{\hbar^{2} c^{2} k^{2}}{m^{*} \rho^{2}}-\frac{\left(\partial_{\rho} \epsilon_{k}\right)^{2}}{m^{*}}-\frac{2 \hbar c k}{\rho}\left(v_{k} \partial_{\rho} c-c \partial_{\rho} v_{k}\right)\right],
\end{aligned}
$$

in agreement with Ref. [30]. Equation (11) has a divergence when $v_{k}$ approaches $c$. At high momentum this occurs after the roton minimum when the quasiparticle becomes supersonic (see Fig. 1). Such a singularity physically represents the possibility for the decay by a single-phonon emission. The same type of divergence in $Y_{k}$ also exists when the quasiparticle approaches the phonon regime. Those two thresholds are the boundaries of validity of our hydrodynamic approach (see the vertical red lines in Fig. 1).

Using the calculated on-shell amplitude, we can now evaluate the damping rate (2). To the leading order in temperature we obtain

$$
\Gamma_{k} \underset{T \rightarrow 0}{\sim} \frac{T^{3} Y_{k}^{2}}{8 \pi \hbar^{3} c^{2} \rho^{2}}\left[\frac{c J\left(\frac{c-v_{k}}{c+v_{k}}\right)}{c+v_{k}}+\frac{c J\left(\frac{c+v_{k}}{c-v_{k}}\right)}{c-v_{k}}\right],
$$

where $Y_{k}$ is given by Eq. (11), while the dimensionless $J(x)=x \int_{0}^{+\infty} d \tilde{q} \tilde{q}^{2}[1+\tilde{n}(x \tilde{q})] \tilde{n}(\tilde{q})$, with $\tilde{n}(\tilde{q})=1 /\left(e^{\tilde{q}}-1\right)$. The rate (12) is our main result. It shows that the lowtemperature quasiparticle decay rate universally scales with the third power of temperature. The expression (12) is general and independent of the particle interaction strength. The $k$ dependent part of the rate is contained in $Y_{k}$ and depends on the specific details of the spectrum. We study now Eq. (12) in more detail.

\section{HARD-PHONON REGIME}

Since $T \ll m c^{2}$, there exists a regime of hard phonons with the characteristic energy $T \ll \epsilon_{k} \ll m c^{2}$ such that our hydrodynamic approach applies. We consider the general form of the spectrum of the energetic quasiparticle, i.e., a hard phonon,

$$
\epsilon_{k}=\hbar c(\rho)|k|\left(1+\frac{\gamma(\rho)}{8} \frac{k^{2}}{q_{0}^{2}}\right)+O\left(k / q_{0}\right)^{4} .
$$

Here the coefficient of the small correction term is negative, $\gamma(\rho)<0$, which ensures that the excitation branch is subsonic (i.e., concave) and that the zero-temperature decay by phonon emission [37] does not occur. Expanding Eq. (11) at small $k$, we obtain $Y_{k}=-\mathcal{A} k / q_{0}+O\left(k / q_{0}\right)^{2}$, where

$$
\mathcal{A}=1-2 \rho \frac{c^{\prime}}{c}-\rho^{2} \frac{c^{\prime 2}}{c^{2}}-\rho^{2} \frac{c^{\prime \prime}}{c}+\rho \frac{\gamma^{\prime}}{\gamma}\left(1+\rho \frac{c^{\prime}}{c}\right) .
$$

The prime denotes the derivative with respect to $\rho$. Using $J(x) \sim 2 \zeta(3) x$ at $x \rightarrow \infty$, the rate (12) becomes

$$
\Gamma_{k} \underset{\substack{T \rightarrow 0 \\ k / q_{0} \rightarrow 0}}{\sim} \frac{32 \zeta(3)}{9 \pi} \mathcal{A}^{2} \frac{q_{0}^{2}}{\gamma^{2} k^{2}} \frac{T^{3}}{\hbar^{3} c^{2} \rho^{2}} .
$$

Equation (15) becomes very large at small $k$, which reflects the denominator in Eq. (11). Our approach requires the quasiparticle energy correction $\delta \epsilon_{k}=\epsilon_{k}-\hbar c|k|$ of Eq. (13) not to be thermally smeared, i.e., $\delta \epsilon_{k} \gg T$. Therefore, the rate (15) applies for phonons of wave vectors satisfying $q_{\mathrm{th}}^{1 / 3} q_{0}^{2 / 3} \ll$ $|k| \ll q_{0}$ with the thermal wave vector $q_{\text {th }}=T / \hbar c$. To study the regime of $k$ comparable to $q_{\text {th }}^{1 / 3} q_{0}^{2 / 3}$, our phonon Hamiltonian (3) should be further expanded to describe four-phonon interactions [31], while the phonon dispersion should now be taken with the correction, as in Eq. (13). In fact, this regime has been studied by a microscopic approach $[41,42]$. For $k=\alpha q_{\mathrm{th}}^{1 / 3} q_{0}^{2 / 3}$ and $\alpha$ independent of temperature, the $T^{3}$ power law for $\Gamma_{k}$ breaks down. However, quite remarkably, one recovers Eq. (15) (specified by the Bogoliubov dispersion, discussed below) by taking the limit $\alpha \rightarrow \infty$ [see Eq. (8) in Ref. [41]]. This excludes the existence of an intermediate scaling law between the regimes $k \approx q_{0}$ [of which Eq. (15) 
gives the low- $k$ limit] and $k \approx q_{\mathrm{th}}^{1 / 3} q_{0}^{2 / 3}$ [of which Eq. (8) in Ref. [41] gives the high- $k$ limit].

\section{CASE OF ROTONS}

Rotons are a special case of the energetic quasiparticles considered in preceding sections, which have a quadratic dispersion around the roton minimum $k_{0}$ :

$$
\epsilon_{k^{\prime}}(\rho)=\Delta(\rho)+\frac{\hbar^{2}\left[k^{\prime}-k_{0}(\rho)\right]^{2}}{2 m^{*}(\rho)}+O\left(k^{\prime}-k_{0}\right)^{3} .
$$

The effective mass $m^{*}$ is positive for rotons, but the present discussion also applies to maxons, which have a similar quadratic dispersion but a negative mass, $m^{*}<0$. For such quadratic dispersion, using $J(1)=\pi^{2} / 3$, the damping rate (12) acquires a simpler form

$$
\Gamma_{k_{0}} \underset{T \rightarrow 0}{\sim} \frac{\pi}{12} Y_{k_{0}}^{2} \frac{T^{3}}{\hbar^{3} c^{2} \rho^{2}},
$$

where one can use Eq. (11) at $k=k_{0}$ or its alternative version

$$
\begin{aligned}
Y_{k_{0}}= & \frac{\rho^{2}}{m c^{2}}\left[\left(\frac{d \mu}{d \rho}\right)^{2} \frac{d^{2} \Delta}{d \mu^{2}}-\frac{d \Delta}{\rho d \rho}-\frac{1}{m_{*}}\left(\frac{d \Delta}{c d \rho}\right)^{2}\right. \\
& \left.+\frac{\hbar^{2}\left(k_{0} / \rho-d k_{0} / d \rho\right)^{2}}{m_{*}}\right]
\end{aligned}
$$

The correspondence with the general form (11) is seen using $\Delta=\epsilon_{k_{0}}, d \Delta / d \rho=\partial_{\rho} \epsilon_{k_{0}}, d^{2} \Delta / d \rho^{2}+\left(\hbar d k_{0} / d \rho\right)^{2} / m^{*}=$ $\partial_{\rho}^{2} \epsilon_{k_{0}}, v_{k_{0}}=0$, and $\hbar d k_{0} / m^{*} d \rho=-\left.\partial_{\rho} v_{k}\right|_{k=k_{0}}$, which follows from partially deriving Eq. (16) with respect to $\rho$ and $k^{\prime}$. The sound velocity $c$ is related to the chemical potential of the gas $\mu$ via the relation $m c^{2}=\rho d \mu / d \rho$.

\section{WEAK-COUPLING LIMIT}

For weak interactions, one can calculate analytically the quasiparticle spectrum [35,37], which takes the Bogoliubov form

$$
\epsilon_{k}=\sqrt{E_{k}\left(E_{k}+2 \rho g_{k}\right)},
$$

where $E_{k}=\hbar^{2} k^{2} / 2 m$ is the kinetic energy of a free boson. Denoted by $g_{k}$ is the Fourier transform of the effective twoparticle interaction. Postponing the discussion about its specific form to the following section, we now assume that the spectrum has the characteristic form of the roton minimum (see Fig. 1). Equation (19) enables us to compute the relevant derivatives with respect to the density:

$$
\begin{aligned}
\rho \partial_{\rho} \epsilon_{k} & =\frac{\epsilon_{k}^{2}-E_{k}^{2}}{2 \epsilon_{k}}, \\
\rho^{2} \partial_{\rho}^{2} \epsilon_{k} & =-\frac{\left(\epsilon_{k}^{2}-E_{k}^{2}\right)^{2}}{4 \epsilon_{k}^{3}}, \\
\rho \partial_{\rho} v_{k} & =v_{k} \frac{\epsilon_{k}^{2}+E_{k}^{2}}{2 \epsilon_{k}^{2}}-\frac{\hbar k}{m} \frac{E_{k}}{\epsilon_{k}} .
\end{aligned}
$$

The roton damping rate (17) now depends only on $\Delta$ and $k_{0}$, besides the thermodynamic parameters $T, c$, and $\rho$, which appeared in Eq. (17). Since those quantities have been measured experimentally, our expression of $\Gamma_{k_{0}}$ can be tested without any assumption about $g_{k}$. In ultracold dipolar bosons the roton gap can be tuned to a wide range of values by tuning the relative strength of the short-range interactions. This suggests that experiments may be more sensitive to the variations of the prefactor of the $T^{3}$ law with $\Delta$ than in superfluid helium (where $\Delta$ can be tuned by varying the pressure but so far in a smaller range). In particular, in the regime $T \ll \Delta \ll E_{k_{0}}, m c^{2}$, we find a divergence of the damping rate as $\Delta^{-6}$.

$$
\Gamma_{k_{0}} \underset{\substack{T \rightarrow 0 \\ \Delta \rightarrow 0}}{\sim} \frac{\pi}{192}(1+R)^{2} \frac{E_{k_{0}}^{8}}{\left(m c^{2}\right)^{2} \Delta^{6}} \frac{T^{3}}{\hbar^{3} c^{2} \rho^{2}} .
$$

In Eq. (21) we have introduced the dimensionless parameter

$$
R=\Delta / m^{*} c^{2}
$$

which has a finite nonzero limit at $\Delta \rightarrow 0$. We finally note that the Bogoliubov spectrum (19) employed in Eq. (14) (in the hard phonon regime) gives $\mathcal{A}=3(\gamma-1) / 2 \gamma$, with $\gamma=$ $1+2 \rho m g_{0}^{\prime \prime} / \hbar^{2}$, which should be substituted in Eq. (15).

\section{EFFECTIVE INTERACTION POTENTIAL}

For the application of our theory to the realistic model, we consider a cylindrically symmetric quasi-one-dimensional Bose gas characterized by the transverse trapping frequency $\omega$ in both transverse directions and the average dipole moment along the direction of motion $x$ [35]. In Fourier space, the effective one-dimensional interaction potential acquires the form

$$
g_{k}=g_{1 \mathrm{D}}-\frac{4 \alpha d^{2}}{l^{2}}\left[1-\check{k}^{2} e^{\check{k}^{2}} \Gamma\left(0, \check{k}^{2}\right)\right],
$$

where $g_{1 \mathrm{D}}$ is the effective one-dimensional contact coupling constant describing short-range interactions (including the short-range behavior of the dipolar interactions $[43,44])$, $\breve{k}=k l / 2$ (with $l=\hbar / \sqrt{m \omega}$ the harmonic-oscillator length), $d$ is the dipole moment, and $\Gamma$ denotes the incomplete Gamma function. The dipoles precess at high frequency around the $x$ axis, which leads to an effective dipolar strength $\alpha d^{2}$ with $-1 / 2<\alpha<1$ [45]. For the interaction potential (23), the sound velocity satisfies $m c^{2}=\rho\left(g_{1 \mathrm{D}}-V_{d}\right)$, where $V_{d}=4 \alpha d^{2} / l^{2}$. The low-energy stability condition requires $g_{1 \mathrm{D}}>V_{d}$. A roton minimum appears at $k_{0}$ (which solves $d \epsilon_{k} /\left.d k\right|_{k=k_{0}}=0$ ) for $g_{1 \mathrm{D}}<0$ and $V_{d, \text { min }}<V_{d}<V_{d, \text { max }}<$ $g_{1 \mathrm{D}}$. The lower bound $V_{d, \min }$ is reached at the phase transition $\Delta=\epsilon_{k_{0}}=0$ and the upper bound $V_{d, \max }$ when the dispersion has an inflection point (when simultaneously $d \epsilon_{k} /\left.d k\right|_{k=k_{0}}=0$ and $d^{2} \epsilon_{k} /\left.d k^{2}\right|_{k=k_{0}}=0$ ).

In Fig. 2 we plot the quasiparticle dispersion and the rescaled damping rate as functions of the wave vector at the interaction strengths $\rho g_{1 \mathrm{D}}=-\hbar \omega$ and $\rho V_{d}=-1.144 \hbar \omega$. The rate $\Gamma_{k}$ diverges at low $k$ as predicted by Eq. (15) as well as at $k_{l} l / 2 \simeq 0.44$ when the excitations become supersonic $\left(v_{k_{l}}=c\right)$. It varies several order of magnitude between the maxon and the roton regimes, the latter being far more 


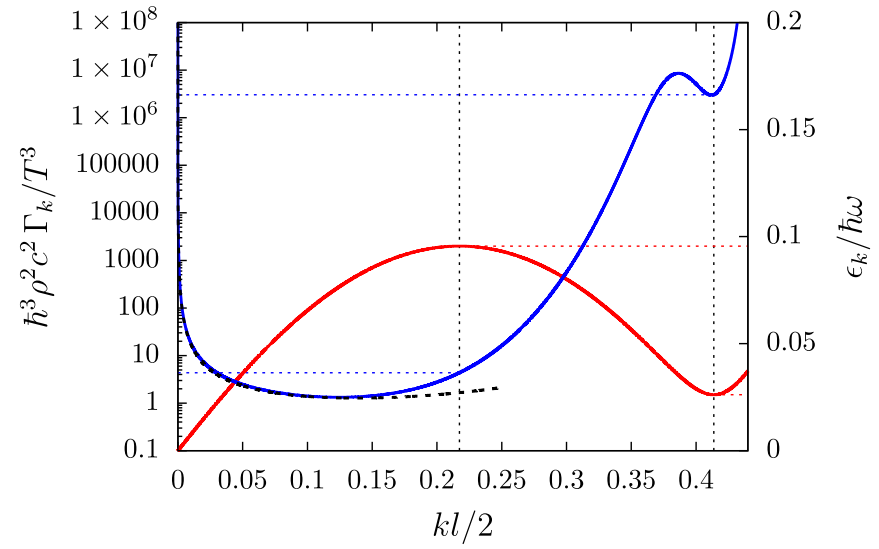

FIG. 2. Quasiparticle spectrum $\epsilon_{k}$ in units of the transverse trapping energy $\hbar \omega$ (red solid line) and rescaled damping rate $\hbar^{3} \rho^{2} c^{2} \Gamma_{k} / T^{3}$ (blue solid line) in a quasi-one-dimensional dipolar Bose gas as functions of the wave vector $k$ in units of $2 / l=$ $2 \sqrt{m \omega} / \hbar$. The dotted vertical black lines show the maxon and roton extrema. The dashed black curve is the hard-phonon asymptote of Eq. (15).

susceptible to the damping. Figure 3 shows the roton damping rate as a function of the dipolar interaction strength $\rho V_{d}$, or equivalently of the roton gap $\Delta$, fixing the short-range coupling constant $\rho g_{1 \mathrm{D}}=-\hbar \omega$. The roton minimum exists for $-1.182 \hbar \omega \lesssim \rho V_{d} \lesssim-1.143 \hbar \omega$ and the gap varies from 0 to about $0.13 \hbar \omega$. The rate $\Gamma_{k}$ is a monotonically decreasing function of $\Delta$ and diverges as $\Delta^{-6}$ when $\Delta \rightarrow 0$ as Eq. (21) predicts.

\section{INFLUENCE OF DIMENSIONALITY}

The $T^{3}$ behavior of the damping rate in one dimension is perhaps our most easily testable prediction. In three dimensions, the correct $T^{7}$ behavior of the roton-phonon damping rate was predicted in [27], although the prefactor is contested by Refs. $[33,40]$. Experimental attempts to observe this decay rate in superfluid helium failed to resolve it from the rotonroton damping rate [46], which follows an activation law in $e^{-\Delta / T}$. In two dimensions, the quasiparticle damping rate of dipolar Bose gas is described in Ref. [47], restricted to Landau-Beliaev-type processes where a quasiparticle decays into two others. This resulted in an exponentially suppressed rate at low temperatures, whereas our theory adapted to the two-dimensional case would give a contribution to the damping rate proportional ${ }^{3}$ to $T^{5}$, hence much larger in the limit $T \rightarrow 0$.

\footnotetext{
${ }^{3}$ This follows from a simple power counting in the two-dimensional equivalent to Eq. (2).
}

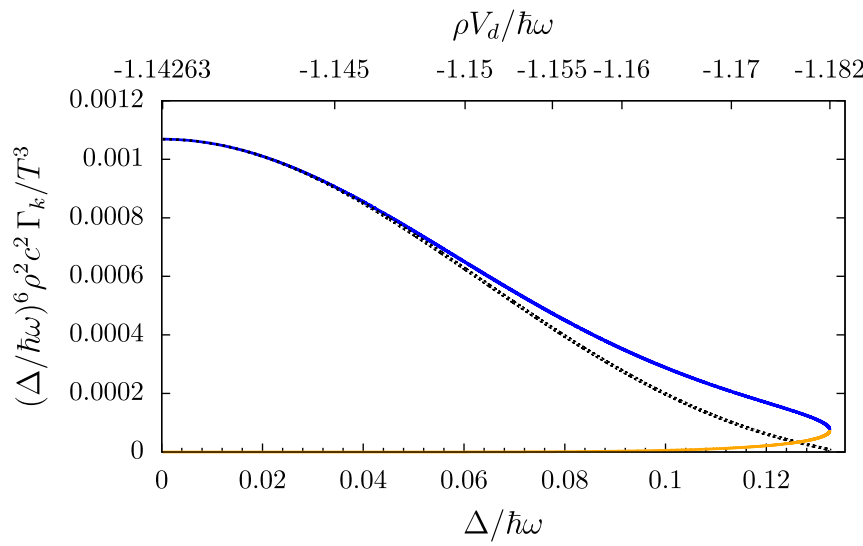

FIG. 3. Rescaled roton (blue curve) and maxon (orange curve) damping rates $(\Delta / \hbar \omega)^{6} \rho^{2} c^{2} \Gamma_{k} / T^{3}$ as functions of the roton gap (lower $x$ axis) or the dipolar strength $\rho V_{d}$ (upper $x$ axis) at fixed $\rho g_{1 \mathrm{D}}=-\hbar \omega$. The dashed black line is the corresponding $\Delta \rightarrow 0$ asymptote of the roton damping rate obtained from Eq. (21).

\section{CONCLUSION AND OUTLOOK}

In this paper we studied the damping of quasiparticles in a dipolar Bose gas comporting rotons. Since the quasiparticles are subsonic, they cannot decay at $T=0$, except at large wave vectors. This should be contrasted with the weakly interacting Bose gas with short-range interactions which has a convex spectrum and thus the decay occurs already at $T=0$ [37]. For weak interactions, the two cases have an overlap at very large wave vectors, where the damping rate at $T=0$ approaches a constant value [36,37].

The method we developed here for one-dimensional dipolar gases can be generalized to higher dimensions as it describes more generally the damping of a high-energy subsonic impurity embedded in the superfluid phonon field. Our approach could thus be used to describe the relaxation of the gapped collective branches of superfluid helium-3 [48] or the low-temperature lifetime of BCS quasiparticles, coupled to an Anderson-Bogoliubov phonon in neutral Fermi superfluids [32] and to lattice phonons in superconductors [49].

\section{ACKNOWLEDGMENTS}

We acknowledge helpful discussions with Y. Bing, L. Chomaz, F. Ferlaino, and G. Natale. This research was supported by the European Union's Horizon 2020 research and innovation program under the Marie Skłodowska-Curie Grant Agreement No. 665501, and in part by the EUR grant NanoX ANR-17-EURE-0009 in the framework of the "Programme des Investissements d'Avenir'.
[1] E. M. Lifshitz and L. P. Pitaevskii, Statistical Physics, Part 2: Theory of the Condensed State (Pergamon, Oxford, 1980).
[2] P. Nozières, Is the roton in superfluid ${ }^{4} \mathrm{He}$ the ghost of a Bragg spot? J. Low Temp. Phys. 137, 45 (2004). 
[3] E. Kim and M. H. W. Chan, Probable observation of a supersolid helium phase, Nature (London) 427, 225 (2004).

[4] J. Day and J. Beamish, Low-temperature shear modulus changes in solid ${ }^{4} \mathrm{He}$ and connection to supersolidity, Nature (London) 450, 853 (2007).

[5] D. Y. Kim and M. H. W. Chan, Absence of Supersolidity in Solid Helium in Porous Vycor Glass, Phys. Rev. Lett. 109, 155301 (2012).

[6] A. Griesmaier, J. Werner, S. Hensler, J. Stuhler, and T. Pfau, Bose-Einstein Condensation of Chromium, Phys. Rev. Lett. 94, 160401 (2005).

[7] Q. Beaufils, R. Chicireanu, T. Zanon, B. Laburthe-Tolra, E. Maréchal, L. Vernac, J.-C. Keller, and O. Gorceix, All-optical production of chromium Bose-Einstein condensates, Phys. Rev. A 77, 061601(R) (2008).

[8] M. Lu, N. Q. Burdick, S. H. Youn, and B. L. Lev, Strongly Dipolar Bose-Einstein Condensate of Dysprosium, Phys. Rev. Lett. 107, 190401 (2011).

[9] K. Aikawa, A. Frisch, M. Mark, S. Baier, A. Rietzler, R. Grimm, and F. Ferlaino, Bose-Einstein Condensation of Erbium, Phys. Rev. Lett. 108, 210401 (2012).

[10] T. Lahaye, C. Menotti, L. Santos, M. Lewenstein, and T. Pfau, The physics of dipolar bosonic quantum gases, Rep. Prog. Phys. 72, 126401 (2009).

[11] M. A. Baranov, M. Dalmonte, G. Pupillo, and P. Zoller, Condensed matter theory of dipolar quantum gases, Chem. Rev. 112, 5012 (2012).

[12] M. Wenzel, F. Böttcher, T. Langen, I. Ferrier-Barbut, and T. Pfau, Striped states in a many-body system of tilted dipoles, Phys. Rev. A 96, 053630 (2017).

[13] I. Ferrier-Barbut, H. Kadau, M. Schmitt, M. Wenzel, and T. Pfau, Observation of Quantum Droplets in a Strongly Dipolar Bose Gas, Phys. Rev. Lett. 116, 215301 (2016).

[14] L. Chomaz, S. Baier, D. Petter, M. J. Mark, F. Wächtler, L. Santos, and F. Ferlaino, Quantum-Fluctuation-Driven Crossover from a Dilute Bose-Einstein Condensate to a Macrodroplet in a Dipolar Quantum Fluid, Phys. Rev. X 6, 041039 (2016).

[15] F. Böttcher, J.-N. Schmidt, M. Wenzel, J. Hertkorn, M. Guo, T. Langen, and T. Pfau, Transient Supersolid Properties in an Array of Dipolar Quantum Droplets, Phys. Rev. X 9, 011051 (2019).

[16] L. Chomaz, D. Petter, P. Ilzhöfer, G. Natale, A. Trautmann, C. Politi, G. Durastante, R. M. W. van Bijnen, A. Patscheider, M. Sohmen, M. J. Mark, and F. Ferlaino, Long-Lived and Transient Supersolid Behaviors in Dipolar Quantum Gases, Phys. Rev. X 9, 021012 (2019).

[17] L. Tanzi, E. Lucioni, F. Famà, J. Catani, A. Fioretti, C. Gabbanini, R. N. Bisset, L. Santos, and G. Modugno, Observation of a Dipolar Quantum Gas with Metastable Supersolid Properties, Phys. Rev. Lett. 122, 130405 (2019).

[18] G. Natale, R. M. W. van Bijnen, A. Patscheider, D. Petter, M. J. Mark, L. Chomaz, and F. Ferlaino, Excitation Spectrum of a Trapped Dipolar Supersolid and its Experimental Evidence, Phys. Rev. Lett. 123, 050402 (2019).

[19] M. Guo, F. Böttcher, J. Hertkorn, J.-N. Schmidt, M. Wenzel, H. P. Büchler, T. Langen, and T. Pfau, The low-energy Goldstone mode in a trapped dipolar supersolid, Nature (London) 574, 386 (2019).
[20] L. Tanzi, S. M. Roccuzzo, E. Lucioni, F. Famà, A. Fioretti, C. Gabbanini, G. Modugno, A. Recati, and S. Stringari, Supersolid symmetry breaking from compressional oscillations in a dipolar quantum gas, Nature (London) 574, 382 (2019).

[21] D. H. J. O'Dell, S. Giovanazzi, and G. Kurizki, Rotons in Gaseous Bose-Einstein Condensates Irradiated by a Laser, Phys. Rev. Lett. 90, 110402 (2003).

[22] L. Santos, G. V. Shlyapnikov, and M. Lewenstein, RotonMaxon Spectrum and Stability of Trapped Dipolar BoseEinstein Condensates, Phys. Rev. Lett. 90, 250403 (2003).

[23] N. Bogolubov, On the theory of superfluidity, J. Phys. USSR 11, 23 (1947).

[24] L. Chomaz, R. M. W. van Bijnen, D. Petter, G. Faraoni, S. Baier, J. H. Becher, M. J. Mark, F. Wächtler, L. Santos, and F. Ferlaino, Observation of roton mode population in a dipolar quantum gas, Nat. Phys. 14, 442 (2018).

[25] P. Ilzhöfer, M. Sohmen, G. Durastante, C. Politi, A. Trautmann, G. Morpurgo, T. Giamarchi, L. Chomaz, M. J. Mark, and F. Ferlaino, Phase coherence in out-of-equilibrium supersolid states of ultracold dipolar atoms, arXiv:1912.10892.

[26] H. Kurkjian, Y. Castin, and A. Sinatra, Brouillage thermique d'un gaz cohérent de fermions, C.R. Phys. 17, 789 (2016).

[27] L. Landau and I. Khalatnikov, Teoriya vyazkosti geliya-II, Zh. Eksp. Teor. Fiz. 19, 637 (1949).

[28] T. Enss, R. Haussmann, and W. Zwerger, Viscosity and scale invariance in the unitary Fermi gas, Ann. Phys. (NY) 326, 770 (2011).

[29] D. M. Gangardt and A. Kamenev, Quantum Decay of Dark Solitons, Phys. Rev. Lett. 104, 190402 (2010).

[30] K. A. Matveev and A. V. Andreev, Scattering of hole excitations in a one-dimensional spinless quantum liquid, Phys. Rev. B 86, 045136 (2012).

[31] H. Kurkjian, Y. Castin, and A. Sinatra, Landau-Khalatnikov phonon damping in strongly interacting Fermi gases, Europhys. Lett. 116, 40002 (2016)

[32] Y. Castin, A. Sinatra, and H. Kurkjian, Landau Phonon-Roton Theory Revisited for Superfluid ${ }^{4} \mathrm{He}$ and Fermi Gases, Phys. Rev. Lett. 119, 260402 (2017).

[33] A. Nicolis and R. Penco, Mutual interactions of phonons, rotons, and gravity, Phys. Rev. B 97, 134516 (2018).

[34] M. Van Regemortel, H. Kurkjian, M. Wouters, and I. Carusotto, Prethermalization to thermalization crossover in a dilute Bose gas following an interaction ramp, Phys. Rev. A 98, 053612 (2018).

[35] S. Sinha and L. Santos, Cold Dipolar Gases in QuasiOne-Dimensional Geometries, Phys. Rev. Lett. 99, 140406 (2007).

[36] S. Tan, M. Pustilnik, and L. I. Glazman, Relaxation of a High-Energy Quasiparticle in a One-Dimensional Bose Gas, Phys. Rev. Lett. 105, 090404 (2010).

[37] Z. Ristivojevic and K. A. Matveev, Decay of Bogoliubov excitations in one-dimensional Bose gases, Phys. Rev. B 94, 024506 (2016).

[38] H. Kurkjian, Y. Castin, and A. Sinatra, Three-phonon and fourphonon interaction processes in a pair-condensed Fermi gas, Ann. Phys. (Berlin) 529, 1600352 (2017).

[39] A. Petković and Z. Ristivojevic, Dynamics of a Mobile Impurity in a One-Dimensional Bose Liquid, Phys. Rev. Lett. 117, 105301 (2016). 
[40] Y. Castin, A. Sinatra, and H. Kurkjian, Erratum: Landau Phonon-Roton Theory Revisited for Superfluid ${ }^{4} \mathrm{He}$ and Fermi Gases [Phys. Rev. Lett. 119, 260402 (2017)], Phys. Rev. Lett. 123, 239904(E) (2019).

[41] J. Lin, K. A. Matveev, and M. Pustilnik, Thermalization of Acoustic Excitations in a Strongly Interacting OneDimensional Quantum Liquid, Phys. Rev. Lett. 110, 016401 (2013).

[42] Z. Ristivojevic and K. A. Matveev, Decay of Bogoliubov quasiparticles in a nonideal one-dimensional Bose gas, Phys. Rev. B 89, 180507(R) (2014).

[43] F. Deuretzbacher, J. C. Cremon, and S. M. Reimann, Ground-state properties of few dipolar bosons in a quasione-dimensional harmonic trap, Phys. Rev. A 81, 063616 (2010).

[44] F. Deuretzbacher, J. C. Cremon, and S. M. Reimann, Erratum: Ground-state properties of few dipolar bosons in a quasi-one- dimensional harmonic trap [Phys. Rev. A 81, 063616 (2010)], Phys. Rev. A 87, 039903(E) (2013).

[45] S. Giovanazzi, A. Görlitz, and T. Pfau, Tuning the Dipolar Interaction in Quantum Gases, Phys. Rev. Lett. 89, 130401 (2002).

[46] B. Fåk, T. Keller, M. E. Zhitomirsky, and A. L. Chernyshev, Roton-Phonon Interactions in Superfluid ${ }^{4} \mathrm{He}$, Phys. Rev. Lett. 109, 155305 (2012).

[47] S. S. Natu and R. M. Wilson, Landau damping in a collisionless dipolar Bose gas, Phys. Rev. A 88, 063638 (2013).

[48] D. Vollhardt and P. Wolfle, The Superfluid Phases of Helium 3 (Taylor \& Francis, London, 1990).

[49] S. B. Kaplan, C. C. Chi, D. N. Langenberg, J. J Chang, S. Jafarey, and D. J. Scalapino, Quasiparticle and phonon lifetimes in superconductors, Phys. Rev. B 14, 4854 (1976). 